\title{
On Exponential Stability For Skew-Evolution Semiflows on Banach SPACES
}

\author{
Codruţa Stoica \\ Institut de Mathématiques \\ Université Bordeaux 1 \\ France \\ e-mail: codruta.stoica@math.u-bordeaux1.fr
}

\begin{abstract}
The paper emphasizes the property of stability for skew-evolution semiflows on Banach spaces, defined by means of evolution semiflows and evolution cocycles and which generalize the concept introduced by us in 10 . There are presented several general characterizations of this asymptotic property out of which can be deduced well known results of the stability theory. A unified treatment in the uniform and in the nonuniform setting is given. The main results are also formulated in discrete time.
\end{abstract}

Mathematics Subject Classification: 34D09

Keywords: Evolution semiflow, evolution cocycle, skew-evolution semiflow, exponential growth, uniform exponential stability, exponential stability, strongly measurable, *-strongly measurable

\section{Preliminaries}

In recent years, an impressive progress concerning the study of asymptotic behaviors for evolution equations can be emphasized, which led to a vast literature due mostly to asymptotic properties of linear operators semigroups. The possibility of reducing the nonautonomous case in the study of evolutionary families or skew-product flows to the autonomous case of evolution semigroups on various Banach function spaces is considered an important way to interesting applications. The study of the asymptotic behavior of linear skew-product semiflows has been used in the theory of evolution equations in infinite dimensional spaces. The approach from point of view of asymptotic properties for the evolution semigroup associated to the linear skew-product semiflows was essential.

Interesting results on the stability of solutions for non autonomous differential equations in Banach spaces were obtained by E.A. Barbashin in 1967 and presented in [1]. One of the most important results of the stability 
theory is due to R. Datko who had proved in 1970 in [4 that a strongly continuous semigroup of operators $\mathcal{S}=\{S(t)\}_{t \geq 0}$ of $\mathcal{C}_{0}$-class defined on a complex Hilbert space $H$ is uniformly exponentially stable if and only if for each vector $x \in H$ the mapping given by $t \rightarrow\|S(t) x\|$ is in $\mathcal{L}^{2}\left(\mathbb{R}_{+}\right)$.

Later, in 1983, A. Pazy generalizes the previous result in [13] for $\mathcal{L}^{p}\left(\mathbb{R}_{+}\right)$, $p \geq 1$, in the case of $\mathcal{C}_{0}$-semigroups in Banach spaces.

Among the remarkable results concerning the property of stability is the classic theorem of R. Datko who proves in 1972 in [5] that a evolutionary process denoted $\mathcal{U}=\{U(t, s)\}_{t \geq s \geq 0}$, with uniform exponential growth, is uniformly exponentially stable if and only if there exists an exponent $p \geq 1$ such that

$$
\sup _{s \geq 0} \int_{s}^{\infty}\|U(t, s) x\|^{p} d t<\infty, \forall x \in V
$$

$V$ being a Banach space.

In 1986, S. Rolewicz presents in [16] a similar characterization for the uniform exponential stability property, proving that if for a continuous nondecreasing mapping $F: \mathbb{R}_{+} \rightarrow \mathbb{R}_{+}$with the properties $F(0)=0$ and $F(t)>0, \forall t>0$ and for an evolutionary process $\mathcal{U}=\{U(t, s)\}_{t \geq s \geq 0}$ on a Banach space $V$, with exponential growth, following relation holds

$$
\sup _{s \geq 0} \int_{s}^{\infty} F(\|U(t, s) x\|) d t<\infty, \forall x \in V,
$$

then the evolutionary process is uniformly exponentially stable.

It was J.M.A.M. van Neerven who, in 1995, referring to the case of a $\mathcal{C}_{0}$-semigroup, proposes in [11] characterizations of the uniform exponential stability in terms of functions spaces. It is proven that a semigroup denoted $\mathcal{S}=\{S(t)\}_{t \geq 0}$ is exponentially stable if there exists a Banach function space $E$ over $\mathbb{R}_{+}$with the property $\lim _{t \rightarrow \infty}\left\|\chi_{[0, t]}\right\|_{E}=\infty$ such that $\|S(\cdot) x\| \in E, \forall x \in V, V$ being a Banach space. The result concerns the evolution semigroup associated to an evolution operator.

In 12 J.M.A.M. van Neerven extends results obtained previously by A. Pazy, S. Rolewicz and R. Datko, as, for instance, in the case of lower semicontinuous functionals. There are presented implications of the fact that a $\mathcal{C}_{0}$-semigroup is not uniformly exponentially stable.

In 2] C. Buşe proves a sufficient condition for the uniform exponential stability of a strongly continuous semigroup on a Banach space, as simplified variant of a theorem of J.M.A.M. van Neerven. A nonautonom version of the theorem for evolution families is also presented and it is emphasized the fact that it implies results obtained by R. Datko, A. Pazy and S. Rolewicz. There can also be emphasized the results obtained by P. Preda, A. Pogan and C. Preda in [15], where it is characterized, from continuous and discrete point of view, the exponential stability of an evolution process in terms of existence of functionals on certain function spaces. 
A generalization of the result of J.M.A.M. van Neerven and characterizations of Perron type for evolution operators were given by C. Preda and P. Preda, who in [14] proves a necessary and sufficient condition for an evolution process of class $\mathcal{C}(0, e)$ to be uniformly exponentially stable and by M. Megan, A.L. Sasu and B. Sasu in [6] evolution operators on Banach spaces, respectively in [9] where there are emphasized discrete and continuous characterizations for the uniform exponential expansiveness of skew-product flows, by means of uniform complete admissibility of the pairs $\left(c_{0}(\mathbb{N}, V), c_{0}(\mathbb{N}, V)\right)$ respectively $\left(\mathcal{C}_{0}\left(\mathbb{R}_{+}, V\right), \mathcal{C}_{0}\left(\mathbb{R}_{+}, V\right)\right), V$ being a Banach space.

In [7, M. Megan, A.L. Sasu and B. Sasu prove necessary and sufficient conditions for the uniform exponential stability of evolution equation on Banach spaces, by emphasizing techniques from the domain of skew-product semiflows and of some function spaces. There are obtained generalizations of some results by R. Datko, S. Rolewicz and J.M.A.M. van Neerven.

In [17, we have studied, among other asymptotic properties, the stability of evolution operators in the uniform setting, by emphasizing relations between the exponential stability and integral stability.

The concept of skew-evolution semiflow, defined by us by means of evolution semiflows and evolution cocycles seems to be more appropriate for the study of asymptotic behaviors of evolution equations by means of evolution operators and also for the nonuniform setting. In fact, we prove that the evolution operators are a particular case of evolution cocycles and that the skew-evolution semiflows are generalizations of the skew-product semiflows.

\section{Definitions and examples}

Let $(X, d)$ be a metric space, $V$ a Banach space, $V^{*}$ its topological dual, $\mathcal{B}(V)$ the space of all $V$-valued bounded operators defined on $V$. We denote $Y=X \times V, T=\left\{\left(t, t_{0}\right) \in \mathbb{R}_{+}^{2}: t \geq t_{0}\right\}$ and $\Delta=\left\{(m, n) \in \mathbb{N}^{2}: m \geq n\right\}$. The norm of vectors on $V$ and on $V^{*}$ and of operators on $\mathcal{B}(V)$ is denoted by $\|\cdot\|$. Let $I$ be the identity operator on $V$. We will consider the set

$$
\mathcal{R}=\left\{R: \mathbb{R}_{+} \rightarrow \mathbb{R}_{+} \mid R \text { nondecreasing, } R(0)=0, R(t)>0, \forall t>0\right\} .
$$

Definition 2.1 A mapping $\varphi: T \times X \rightarrow X$ is called evolution semiflow on $X$ if it satisfies the following properties

$$
\begin{aligned}
& \left(e s_{1}\right) \varphi(t, t, x)=x, \forall(t, x) \in \mathbb{R}_{+} \times X \\
& \left(e s_{2}\right) \varphi\left(t, s, \varphi\left(s, t_{0}, x\right)\right)=\varphi\left(t, t_{0}, x\right), \forall(t, s),\left(s, t_{0}\right) \in T, \forall x \in X .
\end{aligned}
$$

Definition 2.2 A mapping $\Phi: T \times X \rightarrow \mathcal{B}(V)$ that satisfies the following properties

$$
\begin{aligned}
& \left(e c_{1}\right) \Phi(t, t, x)=I, \forall t \geq 0, \forall x \in X \\
& \left(e c_{2}\right) \Phi\left(t, s, \varphi\left(s, t_{0}, x\right)\right) \Phi\left(s, t_{0}, x\right)=\Phi\left(t, t_{0}, x\right), \forall(t, s),\left(s, t_{0}\right) \in T, \forall x \in X
\end{aligned}
$$
is called evolution cocycle over the evolution semiflow $\varphi$. 
Definition 2.3 A mapping $C: T \times Y \rightarrow Y$ defined by

$$
C(t, s, x, v)=(\varphi(t, s, x), \Phi(t, s, x) v), \forall(t, s, x, v) \in T \times Y
$$

where $\Phi$ is an evolution cocycle over the evolution semiflow $\varphi$, is called skew-evolution semiflow on $Y$.

Example 2.1 Let $f: \mathbb{R} \rightarrow \mathbb{R}_{+}$be a function which is nondecreasing on the interval $(-\infty, 0)$ and decreasing on the interval $(0, \infty)$ with the property that there exists

$$
\lim _{t \rightarrow \pm \infty} f(t)=l \in(0, \infty)
$$

Let us consider the set $\mathcal{C}=\mathcal{C}(\mathbb{R}, \mathbb{R})$ of all continuous functions given by $x: \mathbb{R} \rightarrow \mathbb{R}$, endowed with the uniform convergence topology on compact subsets of $\mathbb{R}$. $\mathcal{C}$ is metrizable by respect to the metric

$$
d(x, y)=\sum_{n=1}^{\infty} \frac{1}{2^{n}} \frac{d_{n}(x, y)}{1+d_{n}(x, y)}, \text { where } d_{n}(x, y)=\sup _{t \in[-n, n]}|x(t)-y(t)| .
$$

Let $X$ be the closure of the set $\left\{f_{t}, t \in \mathbb{R}\right\}$ in $\mathcal{C}$, where we consider $f_{t}(\tau)=f(t+\tau), \forall \tau \in \mathbb{R}$. Then $(X, d)$ is a metric space and the mapping

$$
\varphi: T \times X \rightarrow X, \varphi(t, s, x)=x_{t-s}
$$

is an evolution semiflow on $X$.

Let $V=\mathbb{R}^{3}$ be a Banach space with the norm

$$
\left\|\left(v_{1}, v_{2}, v_{3}\right)\right\|=\left|v_{1}\right|+\left|v_{2}\right|+\left|v_{3}\right| .
$$

The mapping $\Phi: T \times X \rightarrow \mathcal{B}(V)$ given by

$\Phi(t, s, x)\left(v_{1}, v_{2}, v_{3}\right)=\left(e^{\alpha_{1} \int_{s}^{t} x(\tau-s) d \tau} v_{1}, e^{-\alpha_{2} \int_{s}^{t} x(\tau-s) d \tau} v_{2}, e^{l+\alpha_{3} \int_{s}^{t} x(\tau-s) d \tau} v_{3}\right)$ where $\left(\alpha_{1}, \alpha_{2}, \alpha_{3}\right) \in \mathbb{R}^{3}$, is an evolution cocycle on $\mathbb{R}^{3}$ and $C=(\varphi, \Phi)$ is a skew-evolution semiflow on $Y$.

Some particular classes of measurable skew-evolution semiflows will be defined in what follows, useful for various characterizations of the property of stability in the uniform as well as in the nonuniform case.

Definition 2.4 A skew-evolution semiflow $C=(\varphi, \Phi)$ is called strongly measurable if for all $\left(t_{0}, x, v\right) \in \mathbb{R}_{+} \times Y$ the mapping

$$
s \mapsto\left\|\Phi\left(s, t_{0}, x\right) v\right\|
$$

is measurable on $\left[t_{0}, \infty\right)$.

Definition 2.5 A skew-evolution semiflow $C=(\varphi, \Phi)$ is called $*$-strongly measurable if for all $\left(t, t_{0}, x, v^{*}\right) \in T \times X \times V^{*}$ the mapping

$$
s \mapsto\left\|\Phi\left(t, s, \varphi\left(s, t_{0}, x\right)\right)^{*} v^{*}\right\|
$$

is measurable on $\left[t_{0}, t\right]$. 


\section{Uniform exponential stability for skew-evolution semiflows}

Some definitions of asymptotic properties in the uniform setting are given in what follows.

Definition 3.1 A skew-evolution semiflow $C=(\varphi, \Phi)$ has uniform exponential growth if there exist some constants $M \geq 1$ and $\omega>0$ such that following relation holds

$$
\left\|\Phi\left(t, t_{0}, x\right) v\right\| \leq M e^{\omega(t-s)}\left\|\Phi\left(s, t_{0}, x\right) v\right\|,
$$

for all $(t, s),\left(s, t_{0}\right) \in T$ and all $(x, v) \in Y$.

Definition 3.2 A skew-evolution semiflow $C=(\varphi, \Phi)$ is called uniformly stable if there exists a constant $N \geq 1$ such that

$$
\left\|\Phi\left(t, t_{0}, x\right) v\right\| \leq N\left\|\Phi\left(s, t_{0}, x\right) v\right\|
$$

for all $(t, s),\left(s, t_{0}\right) \in T$ and all $(x, v) \in Y$.

Definition 3.3 A skew-evolution semiflow $C=(\varphi, \Phi)$ is said to be uniformly exponentially stable if there exist some constants $N \geq 1$ and $\nu>0$ such that

$$
\left\|\Phi\left(t, t_{0}, x\right) v\right\| \leq N e^{-\nu(t-s)}\left\|\Phi\left(s, t_{0}, x\right) v\right\|
$$

for all $(t, s),\left(s, t_{0}\right) \in T$ and all $(x, v) \in Y$.

Example 3.1 We consider $\mathcal{C}=\mathcal{C}\left(\mathbb{R}_{+}, \mathbb{R}\right)$ the set of all continuous functions $x: \mathbb{R}_{+} \rightarrow \mathbb{R}$, with the topology of uniform convergence on bounded sets. Let $f: \mathbb{R}_{+} \rightarrow \mathbb{R}$ be a decreasing function on $[0, \infty)$.

Let $X$ be the closure in $\mathcal{C}$ of the set of functions $f_{\theta}, \theta \in \mathbb{R}_{+}$given by

$$
f_{\theta}(\tau)=f(\theta+\tau), \forall \tau \in \mathbb{R}_{+} .
$$

The mapping

$$
\varphi: T \times X \rightarrow X, \varphi(t, s, x)=x_{t-s}
$$

is an evolution semiflow on the metric space $X$, considered with the metric given in Example 2.1.

We consider the Banach space $V=\mathbb{R}$. The mapping $\Phi: T \times X \rightarrow \mathcal{B}(\mathbb{R})$ given by

$$
\Phi(t, s, x) v=v e^{-\mu(t-s)+\int_{s}^{t} x(\tau-s) d \tau},(t, s) \in T,(x, v) \in X \times \mathbb{R},
$$

where $\mu>x(0)$, is an evolution cocycle over the evolution semiflow $\varphi$.

The skew-evolution semiflow $C=(\varphi, \Phi)$ is uniformly exponentially stable with

$$
N=1 \text { and } \nu=\mu-x(0) \text {. }
$$


Remark 3.1 A uniformly exponentially stable skew-evolution semiflow is uniformly stable which further implies that it has uniform exponential growth.

There exist uniformly stable skew-evolution semiflows which are not uniformly exponentially stable.

Example 3.2 We consider $X=\mathbb{R}_{+}, V=\mathbb{R}$ and a nondecreasing and bounded function $f: \mathbb{R}_{+} \rightarrow \mathbb{R}_{+}^{*}$.

One can easily verify that the mapping $\varphi: T \times \mathbb{R}_{+} \rightarrow \mathbb{R}_{+}$defined by

$$
\varphi(t, s, x)=t-s+x,(t, s, x) \in T \times \mathbb{R}_{+}
$$

is an evolution semiflow on $\mathbb{R}_{+}$and the mapping $\Phi: T \times \mathbb{R}_{+} \rightarrow \mathcal{B}(\mathbb{R})$ given by

$$
\Phi(t, s, x)=\frac{f(x)}{f(t-s+x)},(t, s, x) \in T \times \mathbb{R}_{+}
$$

is an evolution cocycle on $\mathbb{R}$ and $C=(\varphi, \Phi)$ is a uniformly stable skewevolution semiflow, with $N=1$.

On the other hand, if we suppose that there exist some constants $N \geq 1$ and $\nu>0$ such that

$$
\frac{f(x)}{f(t-s+x)} \leq N e^{-\nu(t-s)}, \forall(t, s, x) \in T \times \mathbb{R}_{+} .
$$

Let $s=0$ and we obtain

$$
\frac{e^{\nu t}}{f(t+x)} \leq \frac{N}{f(x)}
$$

which is absurd for $t \rightarrow \infty$. Hence $C$ is not uniformly exponentially stable.

A characterization of the stability property is given by the next result.

Proposition 3.1 A skew-evolution semiflow $C=(\varphi, \Phi)$ is uniformly exponentially stable if and only if there exists a nondecreasing function defined as $f:[0, \infty) \rightarrow(0, \infty)$ with the property $\lim _{t \rightarrow \infty} f(t)=\infty$ such that

$$
f(t-s)\left\|\Phi\left(t, t_{0}, x\right) v\right\| \leq\left\|\Phi\left(s, t_{0}, x\right) v\right\|
$$

for all $(t, s),\left(s, t_{0}\right) \in T$ and all $(x, v) \in Y$.

Proof. Necessity. It is obvious if we consider $f(t)=N^{-1} e^{\nu t}$, where $N$ and $\nu$ are given by Definition 3.3 .

Sufficiency. From the definition of function $f$, there exists $\delta>0$ such that $f(\delta)>1$. We denote

$$
\nu=\frac{\ln f(\delta)}{\delta}>0
$$


Let $(t, s) \in T$. Then there exist $n \in \mathbb{N}$ and $r \in[0, \delta)$ such that $t-s=n \delta+r$. We obtain

$$
\begin{gathered}
e^{\nu(t-s)}\left\|\Phi\left(t, t_{0}, x\right) v\right\| \leq f(\delta)[f(\delta)]^{n}\left\|\Phi\left(t, t_{0}, x\right) v\right\| \leq \\
\leq f(\delta)[f(\delta)]^{n-1}\left\|\Phi\left(t-\delta, t_{0}, x\right) v\right\| \leq \ldots \leq f(\delta)\left\|\Phi\left(t-n \delta, t_{0}, x\right) v\right\| \leq \\
\leq f(\delta) f(r)\left\|\Phi\left(t-n \delta, t_{0}, x\right) v\right\| \leq f(\delta)\left\|\Phi\left(s, t_{0}, x\right) v\right\| .
\end{gathered}
$$

If we consider $N=f(\delta)>1$ it follows that $C$ is uniformly exponentially stable.

Following result can be considered a sufficient condition for a skewevolution semiflow to be uniformly exponentially stable. We will prove a particular case of a result presented by M. Megan, A.L. Sasu and B. Sasu in [8] for evolution families and C. Preda and P. Preda in [14] for evolution processes.

Lemma 3.1 Let $C=(\varphi, \Phi)$ be a skew-evolution semiflow with uniform exponential growth with the property that there exists $\tau>s+1$ such that

$$
\|\Phi(\tau, s, x) v\| \leq \frac{1}{2}, \forall(s, x, v) \in \mathbb{R}_{+} \times Y \text { with }\|v\|=1 .
$$

Then $C$ is uniformly exponentially stable.

Proof. According to the hypothesis there exists $\delta \geq 2$ such that following relation holds

$$
\|\Phi(s+\delta, s, x) v\|<\frac{1}{2}\|v\|, \forall s \geq 0, \forall(x, v) \in Y .
$$

Let us consider $(t, s) \in T$. Then there exist $n \in \mathbb{N}$ and $r \in[0, \delta)$ such that $t=s+n \delta+r$.

We obtain successively

$$
\begin{gathered}
\|\Phi(t, s, x) v\|=\|\Phi(t, s+n \delta, \varphi(s+n \delta, s, x)) \Phi(s+n \delta, s, x) v\| \leq \\
\leq M e^{\omega r}\|\Phi(s+n \delta, s, x) v\| \leq \frac{1}{2} M e^{\omega \delta}\|\Phi(s+(n-1) \delta, s, x) v\| \leq \ldots \leq \\
\leq 2^{-n} M e^{\omega \delta}\|v\|,
\end{gathered}
$$

for all $(t, s) \in T$ and for all $(x, v) \in Y$, where $M$ and $\omega$ are given by means of Definition 3.1.

It follows that

$$
\|\Phi(t, s, x) v\| \leq N e^{-\nu(t-s)}\|v\|, \forall(t, s) \in T, \forall(x, v) \in Y,
$$

where we have denoted

$$
N=M e^{2 \omega \delta} \text { and } \nu=\delta^{-1} \ln 2,
$$

which ends the proof. 
Theorem 3.1 Let $C=(\varphi, \Phi)$ be a strongly measurable skew-evolution semiflow with uniform exponential growth. Following statements are equivalent:

(i) $C$ is uniformly exponentially stable

(ii) there exist a function $R \in \mathcal{R}$ and a constant $N \geq 1$ such that

$$
\int_{t}^{\infty} R\left(\left\|\Phi\left(s, t_{0}, x\right) v\right\|\right) d s \leq N R\left(\left\|\Phi\left(t, t_{0}, x\right) v\right\|\right),
$$

for all $\left(t, t_{0}, x, v\right) \in T \times Y$

(iii) there exist a function $R \in \mathcal{R}$ and a constant $N \geq 1$ such that

$$
\int_{t_{0}}^{\infty} R\left(\left\|\Phi\left(s, t_{0}, x\right) v\right\|\right) d s \leq N R(\|v\|),
$$

for all $\left(t_{0}, x, v\right) \in \mathbb{R}_{+} \times Y$.

Proof. $(i) \Rightarrow(i i)$ It is obtained immediately by means of Definition 3.3 if we consider $R(t)=t, t \geq 0$.

(ii) $\Rightarrow$ (iii) It follows by considering $t=t_{0}$.

$($ iii $) \Rightarrow(i)$ Let $t \geq t_{0}+1$. For $s \in[t-1, t]$ the existence of constants $M \geq 1$ and $\omega>0$ is assured by Definition 3.1 such that

$$
\left\|\Phi\left(t, t_{0}, x\right) v\right\| \leq M e^{\omega}\left\|\Phi\left(s, t_{0}, x\right) v\right\|, \forall(x, v) \in Y
$$

and, further

$$
R\left(\left\|\Phi\left(t, t_{0}, x\right) v\right\|\right) \leq R\left(M e^{\omega}\left\|\Phi\left(s, t_{0}, x\right) v\right\|\right), \forall(x, v) \in Y .
$$

By integrating on $[t-1, t]$ we obtain for all $(x, v) \in Y$

$$
\begin{gathered}
R\left(\left\|\Phi\left(t, t_{0}, x\right) v\right\|\right) \leq \int_{t-1}^{t} R\left(M e^{\omega}\left\|\Phi\left(s, t_{0}, x\right) v\right\|\right) d s \leq \\
\leq \int_{t_{0}}^{\infty} R\left(M e^{\omega}\left\|\Phi\left(s, t_{0}, x\right) v\right\|\right) d s \leq N R\left(M e^{\omega}\|v\|\right) .
\end{gathered}
$$

We have proved

$$
R\left(\left\|\Phi\left(t, t_{0}, x\right) v\right\|\right) \leq N R\left(M e^{\omega}\|v\|\right), \forall t_{0} \geq 0, t \geq t_{0}+1, \forall(x, v) \in Y .
$$

By integrating on $\left[t_{0}+1, t\right]$ it follows

$$
\begin{gathered}
\left(t-t_{0}-1\right) R\left(\left\|\Phi\left(t, t_{0}, x\right) v\right\|\right)= \\
=\int_{t_{0}+1}^{t} R\left(\left\|\Phi\left(t, s, \varphi\left(s, t_{0}, x\right)\right) v\right\|\left\|\Phi\left(s, t_{0}, x\right) v\right\|\right) d s \leq \\
\leq N \int_{t_{0}+1}^{t} R\left(M e^{\omega}\left\|\Phi\left(s, t_{0}, x\right) v\right\|\right) d s \leq N^{2} R\left(M e^{\omega}\|v\|\right) .
\end{gathered}
$$


Hence we have showed

$$
R\left(\left\|\Phi\left(t, t_{0}, x\right) v\right\|\right) \leq \frac{N^{2}}{t-t_{0}-1} R\left(M e^{\omega}\right),
$$

for all $t>t_{0}+1, t_{0} \geq 0$, all $x \in X$ and all $v \in V$ with $\|v\|=1$.

Let $t_{1}>t_{0}+1$ such that

$$
\frac{N^{2}}{t_{1}-t_{0}-1} R\left(M e^{\omega}\right)<R\left(\frac{1}{2}\right)
$$

and, further

$$
\left\|\Phi\left(t_{1}, t_{0}, x\right) v\right\| \leq \frac{1}{2}
$$

for all $x \in X$ and all $v \in V$ with $\|v\|=1$. Hence, according to Lemma 3.1, $C$ is uniformly exponentially stable.

If we take $R(t)=t^{p}, t \geq 0, p>1$ we obtain following result.

Corollary 3.1 A strongly measurable skew-evolution semiflow $C$ with uniform exponential growth is uniformly exponentially stable if and only if there exist some constants $p>1$ and $\widetilde{N} \geq 1$ such that

$$
\int_{t_{0}}^{\infty}\left\|\Phi\left(s, t_{0}, x\right) v\right\|^{p} d s \leq \widetilde{N}^{p}\|v\|^{p}, \forall\left(t, t_{0}, x, v\right) \in T \times Y .
$$

Remark 3.2 $(i)$ For $R(t)=t, t \geq 0$, from Theorem 3.1 is obtained Theorem 11, proved by Datko in 1972 in [5] for the uniform exponential stability in the strong topology of $\mathcal{B}(V)$.

(ii) The case of an evolution operator is presented in 1986 by S. Rolewicz in [16], where it is considered a family of uniformly bounded operators and a nondecreasing function $N$ vanishing in 0 but with no convexity restrictions.

Theorem 3.2 Let $C=(\varphi, \Phi)$ be a skew-evolution semiflow with uniform exponential growth and the property that for all $\left(t_{0}, x, v\right) \in \mathbb{R}_{+} \times Y$ the mappings $s \mapsto\left\|\Phi\left(s, t_{0}, x\right) v\right\|$ and $s \mapsto\left\|\Phi\left(s, t_{0}, x\right)\right\|$ are measurable on $\left[t_{0}, \infty\right)$.

Following statements are equivalent:

(i) $C$ is uniformly exponentially stable

(ii) there exist a function $R \in \mathcal{R}$ and a constant $N \geq 1$ such that

$$
\int_{t}^{\infty} R\left(\left\|\Phi\left(s, t_{0}, x\right)\right\|\right) d s \leq N R\left(\left\|\Phi\left(t, t_{0}, x\right)\right\|\right)
$$

for all $\left(t, t_{0}, x\right) \in T \times X$

(iii) there exists a function $R \in \mathcal{R}$ such that

$$
\int_{t_{0}}^{\infty} R\left(\left\|\Phi\left(s, t_{0}, x\right)\right\|\right) d s<\infty
$$

for all $\left(t_{0}, x\right) \in \mathbb{R}_{+} \times X$. 
Proof. The implication $(i) \Rightarrow(i i)$ follows easily for $R(t)=t, t \geq 0$ and according to Definition 3.3. respectively $(i i) \Rightarrow(i i i)$ is obtained for $t=t_{0}$.

$($ iii $) \Rightarrow(i)$ We have

$$
\begin{gathered}
\int_{t_{0}}^{\infty} R\left(\left\|\Phi\left(s, t_{0}, x\right) v\right\|\right) d s \leq \int_{t_{0}}^{\infty} R\left(\left\|\Phi\left(s, t_{0}, x\right)\right\|\|v\|\right) d s \leq \\
\leq \int_{t_{0}}^{\infty} R\left(\left\|\Phi\left(s, t_{0}, x\right)\right\|\right) d s<\infty
\end{gathered}
$$

for all $\left(t_{0}, x\right) \in \mathbb{R}_{+} \times X$ and $v \in V$ with $\|v\| \leq 1$. It follows according to Theorem 3.1 that $C$ is uniformly exponentially stable.

Theorem 3.3 Let $C=(\varphi, \Phi)$ be a *-strongly measurable skew-evolution semiflow. Following statements are equivalent:

(i) $C$ is uniformly exponentially stable

(ii) $C$ is uniformly stable and there exist a function $R \in \mathcal{R}$ and a constant $\tilde{N} \geq 1$ such that

$$
\int_{t_{0}}^{t} R\left(\left\|\Phi\left(t, s, \varphi\left(s, t_{0}, x\right)\right)^{*} v^{*}\right\|\right) d s \leq R\left(\widetilde{N}\left\|v^{*}\right\|\right),
$$

for all $\left(t, t_{0}, x, v^{*}\right) \in T \times X \times \mathcal{V}^{*}$

(iii) $C$ has uniform exponential growth and there exist a function $R \in \mathcal{R}$ and a constant $\widetilde{N} \geq 1$ such that

$$
\int_{t_{0}}^{t} R\left(\left\|\Phi\left(t, s, \varphi\left(s, t_{0}, x\right)\right)^{*} v^{*}\right\|\right) d s \leq R\left(\tilde{N}\left\|v^{*}\right\|\right),
$$

for all $\left(t, t_{0}, x, v^{*}\right) \in T \times X \times \mathcal{V}^{*}$.

Proof. The implications $(i) \Rightarrow(i i) \Rightarrow($ iii $)$ are obtained by considering $R(t)=t, t \geq 0$, and according to Remark 3.1. We obtain also, as $C$ is uniformly exponentially stable

$$
\int_{t_{0}}^{t}\left\|\Phi\left(t, s, \varphi\left(s, t_{0}, x\right)\right)^{*} v^{*}\right\| d s \leq N \int_{t_{0}}^{t} e^{-\nu(t-s)}\left\|v^{*}\right\| d s \leq \widetilde{N}\left\|v^{*}\right\|,
$$

for all $\left(t, t_{0}, x, v^{*}\right) \in T \times X \times \mathcal{V}^{*}$, where we have denoted

$$
\widetilde{N}=\frac{N}{\nu}
$$

and where the constants $N$ and $\nu$ are given as in Definition 3.3 ,

$($ iii $) \Rightarrow(i)$ As a first step we prove that $C$ is uniformly stable. We consider $t \geq t_{0}+1$ and $s \in\left[t_{0}, t_{0}+1\right)$. Then

$$
R\left(\frac{1}{M} e^{-\omega\left(s-t_{0}\right)}\left|\left\langle v^{*}, \Phi\left(t, t_{0}, x\right) v\right\rangle\right|\right) \leq
$$




$$
\begin{aligned}
& \leq \int_{t_{0}}^{t_{0}+1} R\left(\frac{\left\|\Phi\left(t, \tau, \varphi\left(\tau, t_{0}, x\right)\right)^{*} v^{*}\right\|\left\|\Phi\left(\tau, t_{0}, x\right) v\right\|}{M e^{\omega\left(s-t_{0}\right)}}\right) d \tau \leq \\
& \leq \int_{t_{0}}^{t_{0}+1} R\left(\left\|\Phi\left(t, \tau, \varphi\left(\tau, t_{0}, x\right)\right)^{*} v^{*}\right\|\right) d \tau \leq R\left(\widetilde{N}\left\|v^{*}\right\|\right),
\end{aligned}
$$

where the constants $M$ and $\omega$ are given by Definition 3.1.

Further we obtain

$$
\left\|\Phi\left(t, t_{0}, x\right) v\right\| \leq M e^{\omega\left(s-t_{0}\right)}\|v\| \leq M e^{\omega}\|v\|, \quad \forall t \geq t_{0}+1, \forall x \in X .
$$

For $t \in\left[t_{0}, t_{0}+1\right)$ we have

$$
\left\|\Phi\left(t, t_{0}, x\right)\right\| \leq M e^{\omega\left(t-t_{0}\right)} \leq M e^{\omega}, \forall x \in X .
$$

Hence, $C$ is uniformly stable.

As a second step we prove that $C$ is uniformly exponentially stable. We remark that for all $t_{0} \geq 0$ there exists $t>t_{0}$ such that

$$
\frac{N}{t-t_{0}}<R\left(\frac{1}{2 M}\right) \text {. }
$$

For $t>t_{0}$ it follows that

$$
\begin{gathered}
\left(t-t_{0}\right) R\left(\frac{\left|\left\langle v^{*}, \Phi\left(t, t_{0}, x\right) v\right\rangle\right|}{M}\right) \leq \\
\leq \int_{t_{0}}^{t} R\left(\frac{\left\|\Phi\left(t, s, \varphi\left(s, t_{0}, x\right)\right)^{*} v^{*}\right\|\left\|\Phi\left(s, t_{0}, x\right) v\right\|}{M}\right) d s \leq \\
\leq \int_{t_{0}}^{t} R\left(\left\|\Phi\left(t, s, \varphi\left(s, t_{0}, x\right)\right)^{*} v^{*}\right\|\right) d s \leq N .
\end{gathered}
$$

Hence, for all $t_{0} \geq 0$ there exists $t>t_{0}$ such that

$$
\frac{\left|\left\langle v^{*}, \Phi\left(t, t_{0}, x\right) v\right\rangle\right|}{M} \leq \frac{1}{2 M} .
$$

As $\left\|v^{*}\right\| \leq 1$ we obtain that

$$
\left\|\Phi\left(t, t_{0}, x\right)\right\|<\frac{1}{2}, \forall x \in X .
$$

According to Lemma 3.1, $C$ is uniformly exponentially stable, which ends the proof.

For $R(t)=t^{p}, t \geq 0, p>1$ we obtain following result. 
Corollary $3.2 A$ *-strongly measurable skew-evolution semiflow $C=(\varphi, \Phi)$ with uniform exponential growth is uniformly exponentially stable if and only if there exist some constants $p>1$ and $\widetilde{N} \geq 1$ such that

$$
\int_{t_{0}}^{t}\left\|\Phi\left(t, s, \varphi\left(s, t_{0}, x\right)\right)^{*} v^{*}\right\|^{p} d s \leq \widetilde{N}\left\|v^{*}\right\|^{p},
$$

for all $\left(t, t_{0}, x, v^{*}\right) \in T \times X \times \mathcal{V}^{*}$.

Remark 3.3 For the particular case of exponentially bounded evolution families of bounded operators acting on Banach spaces similar results have been obtain by C. Buşe, M. Megan, M. Prajea and P. Preda in [3].

Theorem 3.4 Let $C=(\varphi, \Phi)$ be a *-strongly measurable skew-evolution semiflow. Following statements are equivalent:

(i) $C$ is uniformly exponentially stable

(ii) $C$ is uniformly stable and there exist a function $R \in \mathcal{R}$ and a constant $\bar{N} \geq 1$ such that

$$
\int_{t_{0}}^{t} R\left(\left\|\Phi\left(t, s, \varphi\left(s, t_{0}, x\right)\right)^{*} v^{*}\right\|\right) d s \leq \bar{N},
$$

for all $\left(t, t_{0}, x, v^{*}\right) \in T \times X \times V^{*}$ with $\left\|v^{*}\right\| \leq 1$.

(iii) $C$ has uniform exponential growth and there exist a function $R \in \mathcal{R}$ and a constant $\bar{N} \geq 1$ such that

$$
\int_{t_{0}}^{t} R\left(\left\|\Phi\left(t, s, \varphi\left(s, t_{0}, x\right)\right)^{*} v^{*}\right\|\right) d s \leq \bar{N},
$$

for all $\left(t, t_{0}, x, v^{*}\right) \in T \times X \times V^{*}$ with $\left\|v^{*}\right\| \leq 1$.

Proof. Necessity. Let us consider $R(t)=t, t \geq 0$. As $C$ is uniformly exponentially stable there exist $N \geq 1$ and $\nu>0$ such that

$$
\begin{gathered}
\int_{t_{0}}^{t}\left\|\Phi\left(t, s, \varphi\left(s, t_{0}, x\right)\right)^{*} v^{*}\right\| d s \leq N \int_{t_{0}}^{t} e^{-\nu(t-s)}\left\|v^{*}\right\| d s= \\
=N \int_{0}^{t-t_{0}} e^{-\nu \tau}\left\|v^{*}\right\| d s \leq \bar{N}
\end{gathered}
$$

for all $t \geq t_{0}+1$ and all $\left(t, t_{0}, x, v^{*}\right) \in T \times X \times V^{*}$ with $\left\|v^{*}\right\| \leq 1$, where we have denoted $\bar{N}=N \nu^{-1}$.

Sufficiency. We will first prove that $C$ is uniformly stable.

Without any loss of generality we can consider that there exists $K>0$ such that $R(K) \geq 1$. 
Let $N \geq 1$. We consider $t \geq t_{0}+N+1$ and $s \in\left[t_{0}, t_{0}+N+1\right)$. As in the proof of Theorem 3.3 we obtain

$$
\begin{gathered}
(N+1) R\left(\frac{1}{M} e^{-\omega\left(s-t_{0}\right)}\left|\left\langle v^{*}, \Phi\left(t, t_{0}, x\right) v\right\rangle\right|\right) \leq \\
\leq \int_{t_{0}}^{t_{0}+N+1} R\left(\left\|\Phi\left(t, \tau, \varphi\left(\tau, t_{0}, x\right)\right)^{*} v^{*}\right\|\right) d \tau \leq N<N+1 \leq(N+1) R(K),
\end{gathered}
$$

where the constants $M$ and $\omega$ are given as in Definition 3.1. By taking supremum over $\|v\| \leq 1$ and $\left\|v^{*}\right\| \leq 1$ we obtain

$$
\left\|\Phi\left(t, t_{0}, x\right)\right\| \leq M K e^{\omega\left(s-t_{0}\right)} \leq M K e^{\omega(N+1)}, \forall t \geq t_{0}+N+1, \forall x \in X .
$$

For $t \in\left[t_{0}, t_{0}+N+1\right)$ we have

$$
\left\|\Phi\left(t, t_{0}, x\right)\right\| \leq M e^{\omega\left(t-t_{0}\right)} \leq M e^{\omega(N+1)}, \forall x \in X
$$

and further

$$
\|\Phi(t, s, x)\| \leq M(1+K) e^{\omega(N+1)}, \forall(t, s, x) \in T \times X,
$$

which shows that $C$ is uniformly stable.

By a similar argumentation as in Theorem 3.3 it follows that $C$ is uniformly exponentially stable.

Remark 3.4 Similar results were obtained by C. Buşe in [2] for functions of type $R \in \mathcal{R}$ supposed to be convex.

Often it is possible to formulate results on the asymptotic behaviors of skew-evolution semiflows in a similar way both for the continuous and the discrete case.

Proposition 3.2 A skew-evolution semiflow $C=(\varphi, \Phi)$ with uniform exponential growth is uniformly exponentially stable if and only if there exist some constants $\widetilde{N} \geq 1$ and $\widetilde{\nu}>0$ such that

$$
\left\|\Phi\left(n, n_{0}, x\right) v\right\| \leq \widetilde{N} e^{-\widetilde{\nu}\left(n-n_{0}\right)}\|v\|, \quad \forall\left(n, n_{0}, x, v\right) \in \Delta \times Y .
$$

Proof. Necessity. It can be easily verified, according to Definition 3.3 ,

Sufficiency. As a first step we will consider $t \geq t_{0}+1$ and we will denote $n=[t]$ and $n_{0}=\left[t_{0}\right]$. This implies that

$$
n \leq t<n+1, n_{0} \leq t_{0}<n_{0}+1, n_{0}+1 \leq n
$$

We obtain successively

$$
\left\|\Phi\left(t, t_{0}, x\right) v\right\|=
$$




$$
\begin{gathered}
=\left\|\Phi\left(t, n, \varphi\left(n, n_{0}+1, x\right)\right) \Phi\left(n, n_{0}+1, \varphi\left(n_{0}+1, t_{0}, x\right)\right) \Phi\left(n_{0}+1, t_{0}, x\right) v\right\| \leq \\
\quad \leq M e^{\omega(t-n)}\left\|\Phi\left(n, n_{0}+1, \varphi\left(n_{0}+1, t_{0}, x\right)\right) \Phi\left(n_{0}+1, t_{0}, x\right) v\right\| \leq \\
\leq M e^{\omega} M e^{\omega\left(n_{0}+1-t_{0}\right)}\left\|\Phi\left(n, n_{0}+1, x\right) v\right\| \leq M^{2} \widetilde{N} e^{2(\omega+\widetilde{\nu})} e^{-\widetilde{\nu}\left(t-t_{0}\right)}\|v\|,
\end{gathered}
$$

for all $(x, v) \in Y$, where the existence of the constants $M$ and $\omega$ is assured by Definition 3.1 .

As a second step, for $t \in\left[t_{0}, t_{0}+1\right)$ we have

$$
\left\|\Phi\left(t, t_{0}, x\right) v\right\| \leq M e^{\omega\left(t-t_{0}\right)}\|v\| \leq M e^{\omega+\widetilde{\nu}} e^{-\widetilde{\nu}\left(t-t_{0}\right)}\|v\|,
$$

for all $(x, v) \in Y$. Hence, we obtain

$$
\left\|\Phi\left(t, t_{0}, x\right) v\right\| \leq\left[M^{2} \widetilde{N} e^{2(\omega+\widetilde{\nu})}+M e^{\omega+\widetilde{\nu}}\right] e^{-\widetilde{\nu}\left(t-t_{0}\right)}\|v\|,
$$

for all $\left(t, t_{0}, x, v\right) \in T \times Y$, which proves the uniform exponential stability of the skew-evolution semiflow $C$.

A similar result as the sufficient condition for stability presented in Lemma 3.1 can be proved in the discrete time setting.

Lemma 3.2 Let $C=(\varphi, \Phi)$ be a skew-evolution semiflow with uniform exponential growth with the property that there exists $n_{0} \in \mathbb{N}^{*}$ such that

$$
\left\|\Phi\left(n+n_{0}, n, x\right)\right\| \leq \frac{1}{2},
$$

for all $n \in \mathbb{N}, \forall x \in X$. Then $C$ is uniformly exponentially stable.

Proof. Let us consider $(m, n) \in \Delta$. There exist $k \in \mathbb{N}$ and $r \in\left\{0,1, \ldots, n_{0}-1\right\}$ such that $m=n+k n_{0}+r$. Hence

$$
\begin{gathered}
\|\Phi(m, n, x)\| \leq \\
\leq\left\|\Phi\left(n+k n_{0}+r, n+k n_{0}, \varphi\left(n+k n_{0}, n, x\right)\right)\right\|\left\|\Phi\left(n+k n_{0}, n, x\right)\right\| \leq \\
\leq 2^{-k} M e^{\omega\left(n_{0}-1\right)} .
\end{gathered}
$$

Hence, we obtain

$$
\|\Phi(m, n, x)\| \leq M e^{\omega\left(n_{0}-1\right)} e^{-n_{0}^{-1}(m-n) \ln 2}, \forall(m, n) \in \Delta, \forall x \in X .
$$

According to Proposition 3.2 we obtain the uniform exponential stability of the skew-evolution semiflow $C$.

Theorem 3.5 A skew-evolution semiflow $C=(\varphi, \Phi)$ with uniform exponential growth is uniformly exponentially stable if and only if there exist a function $R \in \mathcal{R}$ and a constant $\widetilde{M} \geq 1$ such that

$$
\sum_{n=\left[t_{0}\right]+1}^{\infty} R\left(\left\|\Phi\left(n, t_{0}, x\right) v\right\|\right) \leq \widetilde{M} R(\|v\|),
$$

for all $\left(n_{0}, x, v\right) \in \mathbb{N} \times Y$ and for all $t_{0} \geq 0$. 
Proof. Necessity. It follows according to Proposition 3.2 if we consider $R(t)=t, t \geq 0$.

Sufficiency. First, we will consider $t \geq t_{0}+1, t_{0} \geq 0$. We define $n=[t]$ and $n_{0}=\left[t_{0}\right]$. It follows that

$$
\begin{aligned}
& R\left(\left\|\Phi\left(t, t_{0}, x\right) v\right\|\right)=R\left(\left\|\Phi\left(t, n, \varphi\left(n, t_{0}, x\right)\right) \Phi\left(n, t_{0}, x\right) v\right\|\right) \leq \\
& \leq R\left(M e^{\omega(t-n)}\left\|\Phi\left(n, t_{0}, x\right) v\right\|\right) \leq R\left(M e^{\omega}\left\|\Phi\left(n, t_{0}, x\right) v\right\|\right),
\end{aligned}
$$

for all $(x, v) \in Y$, where $M$ and $\omega$ are given by Definition 3.1 .

Further we obtain

$$
\begin{gathered}
\int_{t_{0}+1}^{\infty} R\left(\left\|\Phi\left(t, t_{0}, x\right) v\right\|\right) d t \leq \sum_{n=\left[t_{0}\right]+1}^{\infty} R\left(M e^{\omega}\left\|\Phi\left(n, t_{0}, x\right) v\right\|\right) \leq \\
\leq M \bar{M} e^{\omega} R(\|v\|),
\end{gathered}
$$

for all $(x, v) \in Y$. Then, by Theorem 3.1, there exist $N \geq 1$ and $\nu>0$ such that

$$
\left\|\Phi\left(t, t_{0}, x\right) v\right\| \leq N e^{-\nu\left(t-t_{0}\right)}\|v\|, \forall t \geq t_{0}+1, \forall(x, v) \in Y .
$$

We will consider now that $t \in\left[t_{0}, t_{0}+1\right)$. We have

$$
R\left(\left\|\Phi\left(t, t_{0}, x\right) v\right\|\right) \leq R\left(M e^{\omega\left(t-t_{0}\right)}\|v\|\right) \leq R\left(M e^{\omega}\|v\|\right),(x, v) \in Y .
$$

As a conclusion we obtain the uniform exponential stability of $C$.

If we consider $R(t)=t^{p}, t>0, p>0$ we obtain following result.

Corollary 3.3 A skew-evolution semiflow $C=(\varphi, \Phi)$ with uniform exponential growth is uniformly exponentially stable if and only if there exist $p>0$ and a constant $\widetilde{M} \geq 1$ such that

$$
\left(\sum_{k=n_{0}}^{\infty}\left\|\Phi\left(k, n_{0}, x\right) v\right\|^{p}\right)^{\frac{1}{p}} \leq \widetilde{M}\|v\|,
$$

for all $(n, x, v) \in \mathbb{N} \times Y$.

Another characterization for stability in the discrete time case is the following result.

Theorem 3.6 A skew-evolution semiflow $C=(\varphi, \Phi)$ with uniform exponential growth is uniformly exponentially stable if and only if there exist a function $R \in \mathcal{R}$ and a constant $\widetilde{M} \geq 1$ such that

$$
\sum_{k=n_{0}}^{\infty} R(\|\Phi(n, k, x)\|) \leq \widetilde{M},
$$

for all $\left(n, n_{0}, x\right) \in \Delta \times X$. 
Proof. Necessity. It is obtained from the definition of uniform exponential stability if we consider $R(t)=t, t \geq 0$ and Proposition 3.2.

Sufficiency. According to the hypothesis, for all $\left(k, n_{0}\right) \in \Delta$ we obtain

$$
R\left(\left\|\Phi\left(k, n_{0}, x\right)\right\|\right) \leq \widetilde{M}, \forall x \in X .
$$

Further

$$
\begin{gathered}
\sum_{k=n_{0}}^{n} R\left(\left\|\Phi\left(n, n_{0}, x\right)\right\|\right) \leq \sum_{k=n_{0}}^{n} R\left(\|\Phi(n, k, x)\|\left\|\Phi\left(k, n_{0}, x\right)\right\|\right) \leq \\
\leq \widetilde{M} \sum_{k=n_{0}}^{n} R(\|\Phi(n, k, x)\|) \leq \widetilde{M}^{2}
\end{gathered}
$$

which implies

$$
R\left(\left\|\Phi\left(n, n_{0}, x\right)\right\|\right) \leq \frac{\widetilde{M}^{2}}{n-n_{0}+1}, \forall\left(n, n_{0}, x\right) \in \Delta \times X .
$$

There exists $n_{1}>n_{0}+1$ such that

$$
\frac{\widetilde{M}^{2}}{n_{1}-n_{0}+1} \leq R\left(\frac{1}{2}\right)
$$

We obtain that

$$
\left\|\Phi\left(n_{1}, n_{0}, x\right)\right\| \leq \frac{1}{2}, \text { for } n_{1}>n_{0}+1 \text { and } x \in X .
$$

According to Lemma 3.2, the conclusion is obtained.

For $R(t)=t^{p}, t \geq 0, p>0$ following result is obtained.

Corollary 3.4 A skew-evolution semiflow $C=(\varphi, \Phi)$ with uniform exponential growth is uniformly exponentially stable if and only if there exist $p>0$ and a constant $\widetilde{M} \geq 1$ such that

$$
\left(\sum_{k=n_{0}}^{n}\|\Phi(n, k, x) v\|^{p}\right)^{\frac{1}{p}} \leq \widetilde{M}\|v\|, \forall\left(n, n_{0}, x, v\right) \in \Delta \times Y .
$$

Remark 3.5 In [14, C. Preda and P. Preda have obtained results that characterize the stability of $\mathcal{C}_{0}$-semigroups and of evolution processes in continuous and discrete time. 


\section{Exponential stability for skew-evolution semi- flows}

In what follows the definitions and characterizations of exponential growth and exponential stability is obtained in a more general case, the nonuniform setting. Let $C=(\varphi, \Phi)$ be a skew-evolution semiflow on $Y=X \times V$.

Definition 4.1 The skew-evolution semiflow $C=(\varphi, \Phi)$ has exponential growth if there exist a couple of applications $M, \omega: \mathbb{R}_{+} \rightarrow \mathbb{R}_{+}^{*}$ such that

$$
\left\|\Phi\left(t, t_{0}, x\right) v\right\| \leq M(s) e^{\omega(s)(t-s)}\left\|\Phi\left(s, t_{0}, x\right) v\right\|,
$$

for all $(t, s),\left(s, t_{0}\right) \in T$ and all $(x, v) \in Y$.

Remark 4.1 In some cases in it more interesting to consider $\omega \equiv c, c$ being a constant, without any loss of generality,

Remark 4.2 Let us define for $\alpha \in \mathbb{R}$ the mapping $C_{\alpha}: T \times Y \rightarrow Y$ given by $C_{\alpha}(t, s, x, v)=\left(\varphi(t, s, x), \Phi_{\alpha}(t, s, x) v\right)$, where $\varphi$ is an evolution semiflow on $X$ and

$$
\Phi_{\alpha}\left(t, t_{0}, x\right)=e^{-\alpha\left(t-t_{0}\right)} \Phi\left(t, t_{0}, x\right),\left(t, t_{0}, x\right) \in T \times X,
$$

which verifies the conditions of Definition 2.2. It follows that $C_{\alpha}$ is a skewevolution semiflow.

Sometimes it is useful to characterize the asymptotic properties of $C$ by means of those of $C_{\alpha}$

As next relations hold

$$
\begin{gathered}
\left\|\Phi_{\alpha}\left(t, t_{0}, x\right) v\right\|=e^{-\alpha\left(t-t_{0}\right)}\left\|\Phi\left(t, t_{0}, x\right) v\right\| \leq \\
\leq M\left(t_{0}\right) e^{\left[-\alpha+\omega\left(t_{0}\right)\right]\left(t-t_{0}\right)}\|v\|
\end{gathered}
$$

for all $\left(t, t_{0}, x, v\right) \in T \times Y$, where $M$ and $\omega$ ar given as in Definition 4.1, if follows that $C_{\alpha}$ has also exponential growth.

Definition 4.2 A skew-evolution semiflow $C=(\varphi, \Phi)$ is called stable if there exists a function $N: \mathbb{R}_{+} \rightarrow \mathbb{R}_{+}^{*}$ such that

$$
\left\|\Phi\left(t, t_{0}, x\right) v\right\| \leq N(s)\left\|\Phi\left(s, t_{0}, x\right) v\right\|
$$

for all $(t, s),\left(s, t_{0}\right) \in T$ and all $(x, v) \in Y$

Definition 4.3 A skew-evolution semiflow $C=(\varphi, \Phi)$ is said to be exponentially stable if there exist a constant $\nu>0$ and a mapping $N: \mathbb{R}_{+} \rightarrow \mathbb{R}_{+}^{*}$ such that

$$
\left\|\Phi\left(t, t_{0}, x\right) v\right\| \leq N(s) e^{-\nu(t-s)}\left\|\Phi\left(s, t_{0}, x\right) v\right\|,
$$

for all $(t, s),\left(s, t_{0}\right) \in T$ and all $(x, v) \in Y$. 
Example 4.1 Let $X=\mathbb{R}_{+}$and $V=\mathbb{R}$.

We consider the mapping $\Phi_{f}: T \times \mathbb{R}_{+} \rightarrow \mathcal{B}(\mathbb{R})$ given by

$\Phi(t, s, x) v=v \exp (t \sin t-s \sin s-2 t+2 s),(t, s, x, v) \in T \times \mathbb{R}_{+} \times \mathbb{R}$.

We obtain that $C=(\varphi, \Phi)$ is a skew-evolution semiflow on $Y=\mathbb{R}_{+} \times \mathbb{R}$ over every evolution semiflow $\varphi: T \times \mathbb{R}_{+} \rightarrow \mathbb{R}$.

As

$$
t \sin t-s \sin s-2 t+2 s \leq-t+3 s, \forall(t, s) \in T
$$

it follows that

$$
|\Phi(t, s, x) v| \leq e^{2 s} e^{-(t-s)}|v|, \quad \forall(t, s, x, v) \in T \times Y,
$$

and, further, that $C$ is exponentially stable.

In what follows, an example of exponentially stable skew-evolution semiflows which are not uniformly exponentially stable will be emphasized.

Example 4.2 Let us consider $X=\mathbb{R}_{+}$and $V=\mathbb{R}$.

We define the continuous function $f: \mathbb{R}_{+} \rightarrow[1, \infty)$ given by

$$
f(n)=e^{2 n} \text { and } f\left(n+\frac{1}{e^{n^{2}}}\right)=1 .
$$

Let us consider the mapping $\Phi_{f}: T \times \mathbb{R}_{+} \rightarrow \mathcal{B}(\mathbb{R})$ defined by

$$
\Phi_{f}(t, s, x) v=\frac{f(s)}{f(t)} e^{-(t-s)} v,(t, s, x, v) \in T \times \mathbb{R}_{+} \times \mathbb{R} .
$$

Then $C_{f}=\left(\varphi, \Phi_{f}\right)$ is a skew-evolution semiflow on $Y=\mathbb{R}_{+} \times \mathbb{R}$ over any evolution semiflow $\varphi$ pe $\mathbb{R}_{+}$.

As

$$
\left|\Phi_{f}(t, s, x) v\right| \leq f(s) e^{-(t-s)}|v|, \forall(t, s, x, v) \in T \times Y,
$$

we obtain that $C_{f}$ is exponentially stable.

On the other hand, as

$$
\Phi_{f}\left(n+\frac{1}{e^{n^{2}}}, n, x\right)=\exp \left(2 n-\frac{1}{e^{n^{2}}}\right) \rightarrow \infty \text { for } n \rightarrow \infty,
$$

it follows that $C_{f}$ is not uniformly exponentially stable.

The next result is a characterization for the property of exponential stability of skew-evolution semiflows. 
Proposition 4.1 A skew-evolution semiflow $C=(\varphi, \Phi)$ is exponentially stable if and only if there exist a mapping $M:[0, \infty) \rightarrow(0, \infty)$ and a decreasing function $g:[0, \infty) \rightarrow(0, \infty)$ with the property $\lim _{t \rightarrow \infty} g(t)=0$ such that

$$
\left\|\Phi\left(t, t_{0}, x\right) v\right\| \leq M(s) g(t-s)\left\|\Phi\left(s, t_{0}, x\right) v\right\|
$$

for all $(t, s),,\left(s, t_{0}\right) \in T$ and all $(x, v) \in Y$.

Proof. Necessity. It can be easily proven if we consider

$$
M(u)=N(u), u \geq 0 \text { and } g(v)=e^{-\nu v}, v \geq 0,
$$

where $N$ and $\nu$ are given as in Definition 4.3 .

Sufficiency. As $\lim _{t \rightarrow \infty} g(t)=0$, there exists $\delta \in(0,1)$ such that $g(\delta)<1$. Let $(t, s) \in T$. Then there exist $n \in \mathbb{N}$ and $r \in[0, \delta)$ such that $t=s+n \delta+r$. We obtain successively

$$
\begin{gathered}
\left\|\Phi\left(t, t_{0}, x\right) v\right\|=\left\|\Phi\left(s+n \delta+r, t_{0}, x\right) v\right\| \leq \\
\leq g(r) M(s+n \delta)\left\|\Phi\left(s+n \delta, t_{0}, x\right) v\right\| \leq g(0) M(s+n \delta)\left\|\Phi\left(s+n \delta, t_{0}, x\right) v\right\| \leq \\
\leq g(0) g(\delta) M(s+(n-1) \delta+r)\left\|\Phi\left(s+(n-1) \delta, t_{0}, x\right) v\right\| \leq \ldots \leq \\
\leq g(0)[g(\delta)]^{n} M(s+(n-1) \delta) \ldots M(s)\left\|\Phi\left(s+r, t_{0}, x\right) v\right\|,
\end{gathered}
$$

for all $(t, s),\left(s, t_{0}\right) \in T$ and all $(x, v) \in Y$.

If we define

$$
N(u)=\frac{g(0)}{g(\delta)} M(u+(n-1) \delta+r) \ldots M(u+r) M(u) \text { and } \nu=\frac{1}{\delta} \ln \frac{1}{g(\delta)}
$$

we obtain that $C$ is exponentially stable.

Theorem 4.1 Let $C=(\varphi, \Phi)$ be a skew-evolution semiflow with exponential growth. Following statements are equivalent:

(i) $C$ is exponentially stable

(ii) there exist a function $R \in \mathcal{R}$, a function $\tilde{N}:[0, \infty) \rightarrow(0, \infty)$ and a constant $\alpha>0$ such that

$$
\int_{t}^{\infty} R\left(e^{\alpha(s-t)}\left\|\Phi\left(s, t_{0}, x\right) v\right\|\right) d s \leq \tilde{N}(t) R\left(\left\|\Phi\left(t, t_{0}, x\right) v\right\|\right),
$$

for all $\left(t, t_{0}, x, v\right) \in T \times Y$

(iii) there exist a function $R \in \mathcal{R}$, a function $\widetilde{N}:[0, \infty) \rightarrow(0, \infty)$ and a constant $\alpha>0$ such that

$$
\int_{t_{0}}^{\infty} R\left(e^{\alpha\left(s-t_{0}\right)}\left\|\Phi\left(s, t_{0}, x\right) v\right\|\right) d s \leq \tilde{N}\left(t_{0}\right) R(\|v\|),
$$

for all $\left(t_{0}, x, v\right) \in \mathbb{R}_{+} \times Y$. 
Proof. $(i) \Rightarrow(i i)$ We consider $R(t)=t, t \geq 0$ and let us define

$$
\alpha=\frac{\nu}{2}>0
$$

$\nu>0$ being given by the fact that $C$ is exponentially stable.

We obtain successively for all $\left(t, t_{0}\right) \in T$ and $(x, v) \in Y$

$$
\begin{aligned}
\int_{t}^{\infty} e^{\alpha(\tau-t)}\left\|\Phi\left(\tau, t_{0}, x\right) v\right\| d \tau & \leq \int_{t}^{\infty} N(t) e^{\frac{\nu}{2}(\tau-t)} e^{-\nu(\tau-t)}\left\|\Phi\left(t, t_{0}, x\right) v\right\| d \tau= \\
=\widetilde{N}(t)\left\|\Phi\left(t, t_{0}, x\right) v\right\| &
\end{aligned}
$$

where we have denote

$$
\widetilde{N}(t)=\frac{N(t)}{\alpha}, t \geq 0,
$$

the existence of function $N:[0, \infty) \rightarrow(0, \infty)$ being given by Definition 4.3 ,

(ii) $\Rightarrow\left(\right.$ iii) It is obtained by considering $t=t_{0}$.

(iii) $\Rightarrow($ i $)$ First, we will prove that the relation in the hypothesis implies the fact that $C$ is stable. By a similar proof as in the Sufficiency of Theorem 3.1, we obtain that

$$
\left\|\Phi\left(t, t_{0}, x\right) v\right\| \leq M\left(t_{0}\right) N\left(t_{0}\right) K e^{\omega\left(t_{0}\right)}, \forall t \geq t_{0}+1, \forall(x, v) \in Y,
$$

where functions $M$ and $\omega$ are given as in Definition 4.1 and $K$ is chosen such that $R(K) \geq 1$.

Equally, as $C$ has exponential growth, we have

$$
\left\|\Phi\left(t, t_{0}, x\right) v\right\| \leq M\left(t_{0}\right) e^{\omega\left(t_{0}\right)}, \forall t \in\left[t_{0}, t_{0}+1\right), \forall(x, v) \in Y .
$$

Hence we obtain

$$
\left\|\Phi\left(t, t_{0}, x\right) v\right\| \leq M\left(t_{0}\right)\left[1+K N\left(t_{0}\right)\right] e^{\omega\left(t_{0}\right)}, \forall\left(t, t_{0}\right) \in T, \forall(x, v) \in Y,
$$

which proves the stability of $C$.

As a second step we will prove the exponential stability of $C$.

According to the relation in the hypothesis, we obtain that $C_{-\alpha}$ is stable, where $C_{-\alpha}=\left(\varphi, \Phi_{-\alpha}\right)$ is given as in Remark4.2, which assures the existence of a function $\widetilde{M}: \mathbb{R}_{+} \rightarrow \mathbb{R}_{+}^{*}$ such that

$$
e^{\alpha\left(t-t_{0}\right)}\left\|\Phi\left(t, t_{0}, x\right) v\right\| \leq \widetilde{M}\left(t_{0}\right)\|v\|, \forall\left(t, t_{0}, x, v\right) \in T \times Y,
$$

which proves the exponential stability of the skew-evolution semiflow $C$ and ends the proof.

Remark 4.3 For $R(t)=t, t \geq 0$ we obtain a generalization in the nonuniform setting of the classic result proved in Theorem 11 of [5]. 
Theorem 4.2 Let $C=(\varphi, \Phi)$ be a skew-evolution semiflow with exponential growth. Following statements are equivalent:

(i) $C$ is exponentially stable

(ii) there exist a function $R \in \mathcal{R}$, a function $\tilde{N}:[0, \infty) \rightarrow(0, \infty)$ and a constant $\alpha>0$ such that

$$
\int_{t}^{\infty} R\left(e^{\alpha(s-t)}\left\|\Phi\left(s, t_{0}, x\right)\right\|\right) d s \leq \widetilde{N}(t) R\left(\left\|\Phi\left(t, t_{0}, x\right)\right\|\right),
$$

for all $\left(t, t_{0}, x\right) \in T \times X$

(iii) there exist a function $R \in \mathcal{R}$ and a constant $\alpha>0$ such that

$$
\int_{t_{0}}^{\infty} R\left(e^{\alpha\left(s-t_{0}\right)}\left\|\Phi\left(s, t_{0}, x\right)\right\|\right) d s<R\left(t_{0}\right),
$$

for all $\left(t_{0}, x\right) \in \mathbb{R}_{+} \times X$.

Proof. The implications $(i) \Rightarrow(i i) \Rightarrow($ iii $)$ follows easily if we consider $R(t)=t, t \geq 0$.

$($ iii $) \Rightarrow(i)$ As following relation hold

$$
\begin{gathered}
\int_{t_{0}}^{\infty} R\left(e^{\alpha\left(s-t_{0}\right)}\left\|\Phi\left(s, t_{0}, x\right) v\right\|\right) d s \leq \\
\leq\|v\| \int_{t_{0}}^{\infty} R\left(e^{\alpha\left(s-t_{0}\right)}\left\|\Phi\left(s, t_{0}, x\right)\right\|\|v\|\right) d s<R\left(t_{0}\right)
\end{gathered}
$$

for all $\left(t_{0}, x, v\right) \in \mathbb{R}_{+} \times Y$ with $\|v\| \leq 1$, it results by Theorem 4.1 that $C$ is exponentially stable.

Theorem 4.3 Let $C=(\varphi, \Phi)$ be a skew-evolution semiflow with exponential growth. $C$ is exponentially stable if and only if there exist a function $R \in \mathcal{R}$, a mapping $N: \mathbb{R}_{+} \rightarrow[1, \infty)$ and a constant $\alpha>0$ such that

$$
\int_{t_{0}}^{t} R\left(e^{\alpha(t-s)}\left\|\Phi\left(t, s, \varphi\left(s, t_{0}, x\right)\right)^{*} v^{*}\right\|\right) d s \leq N\left(t_{0}\right),
$$

for all $\left(t, t_{0}, x, v^{*}\right) \in T \times X \times V^{*}$ with $\left\|v^{*}\right\| \leq 1$.

Proof. Necessity. If we consider $R(t)=t, t \geq 0$ and if we define $\alpha=-\frac{\omega}{2}$ we obtain

$$
\int_{t_{0}}^{t} e^{\alpha(t-s)}\left\|\Phi\left(t, s, \varphi\left(s, t_{0}, x\right)\right)^{*} v^{*}\right\| d s \leq M\left(t_{0}\right) \int_{t_{0}}^{t} e^{\frac{\omega}{2}(t-s)}\left\|v^{*}\right\| d s \leq N\left(t_{0}\right),
$$

for all $\left(t, t_{0}, x, v^{*}\right) \in T \times X \times V^{*}$ with $\left\|v^{*}\right\| \leq 1$, where we have defined

$$
N(u)=\frac{2}{\omega} M(u),
$$


function $M$ given by the exponential growth of $C$.

Sufficiency. Let us consider $t \geq t_{0}+N\left(t_{0}\right)+1$. We have

$$
\begin{gathered}
{\left[N\left(t_{0}\right)+1\right] R\left(\frac{e^{\alpha\left(t-t_{0}\right)} e^{-\omega\left(t_{0}\right)\left(s-t_{0}\right)}}{M\left(t_{0}\right)}\left|\left\langle v^{*}, \Phi\left(t, t_{0}, x\right) v\right\rangle\right|\right)} \\
\leq \int_{t_{0}}^{t_{0}+N\left(t_{0}\right)+1} R\left(\frac{e^{\alpha(t-s)}\left\|\Phi\left(t, s, \varphi\left(s, t_{0}, x\right)\right)^{*} v^{*}\right\|\left\|\Phi\left(s, t_{0}, x\right) v\right\|}{M\left(t_{0}\right) e^{\omega\left(t_{0}\right)\left(s-t_{0}\right)} e^{-\alpha\left(s-t_{0}\right)}}\right) d s \leq \\
\leq \int_{t_{0}}^{t_{0}+N\left(t_{0}\right)+1} R\left(e^{\alpha(t-s)}\left\|\Phi\left(t, s, \varphi\left(s, t_{0}, x\right)\right)^{*} v^{*}\right\|\right) d s \leq \\
\leq N\left(t_{0}\right)<\left[N\left(t_{0}\right)+1\right] R(1),
\end{gathered}
$$

where functions $M$ and $\omega$ are assured by Definition 4.1 and where, without loss of generality, we have supposed $R(1)>1$. By taking supremum over $\|v\| \leq 1$ and $\left\|v^{*}\right\| \leq 1$ we obtain

$$
e^{\alpha\left(t-t_{0}\right)}\left\|\Phi\left(t, t_{0}, x\right)\right\| \leq M\left(t_{0}\right) e^{\omega\left(t_{0}\right)\left(s-t_{0}\right)} \leq M\left(t_{0}\right) e^{\omega\left(t_{0}\right)\left[N\left(t_{0}\right)+1\right]},
$$

for all $t \geq t_{0}+N\left(t_{0}\right)+1$ and all $x \in X$.

If we take $t \in\left[t_{0}, t_{0}+N\left(t_{0}\right)+1\right)$ we have

$$
\left\|\Phi\left(t, t_{0}, x\right)\right\| \leq M\left(t_{0}\right) e^{\left[\omega\left(t_{0}\right)-\alpha\right]\left(t-t_{0}\right)} \leq M\left(t_{0}\right) e^{\left[\omega\left(t_{0}\right)-\alpha\right]\left[N\left(t_{0}\right)+1\right]}
$$

for all $x \in X$.

It follows that $C_{-\alpha}$ is stable, where $C_{-\alpha}=\left(\varphi, \Phi_{-\alpha}\right)$ is given as in Remark 4.2. Then there exists a function $\tilde{N}: \mathbb{R}_{+} \rightarrow[1, \infty)$ such that

$$
e^{\alpha\left(t-t_{0}\right)}\left\|\Phi\left(t, t_{0}, x\right)\right\| \leq \widetilde{N}\left(t_{0}\right), \forall\left(t, t_{0}, x\right) \in T \times X,
$$

which, as $\alpha>0$ implies the exponential stability of the skew-evolution semiflow $C$.

As in the uniform setting, we can describe asymptotic properties of skewevolution semiflows, as stability, in the discrete time case. Such a characterization for the exponential stability is emphasized in the next

Proposition 4.2 A skew-evolution semiflow $C=(\varphi, \Phi)$ with exponential growth is exponentially stable if and only if there exist a constant $\mu>0$ and a sequence of real numbers $\left(a_{n}\right)_{n \geq 0}$ with the property $a_{n} \geq 1, \forall n \geq 0$ such that

$$
\left\|\Phi\left(n, n_{0}, x\right) v\right\| \leq a_{n} e^{-\mu\left(n-n_{0}\right)}\|v\|,
$$

for all $\left(n, n_{0}, x, v\right) \in \Delta \times Y$. 
Proof. Necessity. It can be easily shown.

Sufficiency. If $t \geq t_{0}+1$ and $n=[t]$ and $n_{0}=\left[t_{0}\right]$ we have

$$
n \leq t<n+1, n_{0} \leq t_{0}<n_{0}+1, n_{0}+1 \leq n
$$

We obtain

$$
\begin{gathered}
\left\|\Phi\left(t, t_{0}, x\right) v\right\| \leq \\
\leq M(n) e^{\omega(n)(t-n)}\left\|\Phi\left(n, n_{0}+1, \varphi\left(n_{0}+1, t_{0}, x\right)\right) \Phi\left(n_{0}+1, t_{0}, x\right) v\right\| \leq \\
\leq a_{n} M^{2}(n) e^{2[\omega(n)+\mu]} e^{-\mu\left(t-t_{0}\right)}\|v\|,
\end{gathered}
$$

for all $(x, v) \in Y$, where the existence of the functions $M$ and $\omega$ is assured by Definition 4.1 .

As a second step, for $t \in\left[t_{0}, t_{0}+1\right)$ we have

$$
\left\|\Phi\left(t, t_{0}, x\right) v\right\| \leq M\left(t_{0}\right) e^{\omega\left(t_{0}\right)\left(t-t_{0}\right)}\|v\| \leq M\left(t_{0}\right) e^{\omega\left(t_{0}\right)+\mu} e^{-\mu\left(t-t_{0}\right)}\|v\|,
$$

for all $(x, v) \in Y$.

Hence, we obtain the exponential stability of $C$.

Some characterization for the stability of skew-evolution semiflows in the nonuniform setting similar to the continuous case are presented in discrete time.

Theorem 4.4 A skew-evolution semiflow $C=(\varphi, \Phi)$ is exponentially stable if and only if there exist a function $R \in \mathcal{R}$, a constant $\rho>0$ and a sequence of real numbers $(\alpha n)_{n \geq 0}$ with the property $\alpha_{n} \geq 1, \forall n \geq 0$ such that

$$
\sum_{k=n}^{m} R\left(e^{\rho(k-n)}\|\Phi(k, n, x) v\|\right) \leq \alpha_{n} R(\|v\|)
$$

for all $(m, n, x, v) \in \Delta \times Y$.

Proof. Necessity. Let us take $R(t)=t, t \geq 0$. Definition 4.3 provides the constant $\nu>0$ and the sequence of real numbers $\left(a_{n}\right)_{n \geq 0}$ with the property $a_{n} \geq 1, \forall n \geq 0$. We obtain for $\rho=\frac{\nu}{2}>0$ and according to Proposition 4.2

$$
\begin{gathered}
\sum_{k=n}^{m} e^{\rho(k-n)}\|\Phi(k, n, x) v\| \leq a_{n} \sum_{k=n}^{m} e^{\rho(k-n)} e^{-\nu(k-n)}\|\Phi(n, n, x) v\|= \\
=a_{n}\|v\| \sum_{k=n}^{m} e^{-\frac{\nu}{2}(k-n)} \leq \alpha_{n}\|v\|, \forall m, n \in \Delta, \forall(x, v) \in Y,
\end{gathered}
$$

where we have denoted

$$
\alpha_{n}=a_{n} e^{\frac{\nu}{2}}, n \in \mathbb{N} \text {. }
$$


Sufficiency. According to the hypothesis and by a similar proof as in Theorem 3.5 we have that $C_{-\rho}=\left(\varphi, \Phi_{-\rho}\right)$ is stable, where

$$
\Phi_{-\rho}(m, n, x)=e^{\rho(m-n)}\|\Phi(m, n, x)\|,(m, n, x) \in \Delta \times X .
$$

Thus there exists a sequence of real numbers $\left(a_{n}\right)_{n \geq 0}$ with the property $a_{n} \geq 1, \forall n \geq 0$, such that

$$
e^{\rho(m-n)}\|\Phi(m, n, x) v\| \leq a_{n}\|v\|, \forall(m, n, x, v) \in \Delta \times Y,
$$

which implies the exponential stability of the skew-evolution semiflow $C$ and ends the proof.

Theorem 4.5 A skew-evolution semiflow $C$ is exponentially stable if and only if there exist a function $R \in \mathcal{R}$, a constant $\gamma>0$ and a sequence of real numbers $(\beta n)_{n \geq 0}$ with the property $\beta_{n} \geq 1, \forall n \geq 0$ such that

$$
\sum_{k=n}^{m} R\left(e^{\gamma(m-k)}\left\|\Phi(m, k, \varphi(k, n, x))^{*} v^{*}\right\|\right) \leq \beta_{n} R\left(\left\|v^{*}\right\|\right)
$$

for all $(m, n, x, v) \in \Delta \times Y$.

Proof. Necessity. For $R(t)=t, t \geq 0$ and $\gamma=\frac{\nu}{2}>0$ we obtain according to the definition of stability and Proposition 4.2

$$
\sum_{k=n}^{m} e^{\frac{\nu}{2}(m-k)}\left\|\Phi(m, k, \varphi(k, n, x))^{*} v^{*}\right\| \leq a_{n}\left\|v^{*}\right\| \sum_{k=n}^{m} e^{-\frac{\nu}{2}(m-k)} \leq \beta_{n}\left\|v^{*}\right\|
$$

where we have denoted

$$
\beta_{n}=\frac{a_{n}}{1-e^{-\frac{\nu}{2}}}, n \in \mathbb{N},
$$

where the constant $\nu>0$ and the sequence of real numbers $\left(a_{n}\right)_{n \geq 0}$ with the property $a_{n} \geq 1, \forall n \geq 0$ are given by Definition 4.3 .

Sufficiency. According to the hypothesis and by a similar proof as in Theorem 3.6 we have that $C_{-\gamma}=\left(\varphi, \Phi_{-\gamma}\right)$ given as in Remark 4.2 is stable. It follows as in the proof of Theorem 4.4 that the skew-evolution semiflow $C$ is exponentially stable.

\section{References}

[1] E.A. Barbashin, Introduction dans la theorie de la stabilité, Izd. Nauka, Moscou (1967)

[2] C. Buşe, Comportări asimptotice ale proceselor evolutive, Ed. Sedona, Timişoara (1998) 
[3] C. Buşe, M. Megan, M. Prajea, P. Preda, The strong variant of a Barbashin theorem on stability of solutions for nonautonomous differential equations in Banach spaces, Integral Equations Operator Theory, DOI 10.1007/s00020-007-1533-x

[4] R. DAtKo, Extending a theorem of A.M. Liapunov to Hilbert space, J. Math. Anal. Appl. 32 (1970) 610-616

[5] R. DAтKO, Uniform asymptotic stability of evolutionary processes in Banach spaces, Siam J. Math. Anal. 3 (1972) 428-445

[6] M. Megan, A.L. Sasu, B. Sasu, Theorems of Perron type for evolution operators, Rend. Mat. Appl. (7) 21 (2001) 231-244

[7] M. Megan, A.L. SASu, B. SASu, On uniform exponential stability of linear skewproduct semiflows in Banach spaces, Bull. Belg. Math. Soc. Simon Stevin 9 (2002) $143-154$

[8] M. Megan, A.L. Sasu, B. Sasu, Asymptotic Behaviour of Evolution Families, Ed. Mirton (2003)

[9] M. Megan, A.L. Sasu, B. Sasu, Perron conditions for uniform exponential expansiveness of linear skew-product flows, Monatsh. Math. 138 (2003) 145-157

[10] M. Megan, C. Stoica, L. Buliga, On asymptotic behaviors for linear skewevolution semiflows in Banach spaces, Carpathian J. Math. 23 (2007) 117-125

[11] J.M.A.M. VAN NEERVEN, Exponential stability of operators and operator semigroups, J. Funct. Anal. 130 No.2 (1995) 293-309

[12] J.M.A.M. VAn NeERVEn, Lower semicontinuity and the theorems of Datko and Pazy, Integral Equations Operator Theory 42 (2002) 482-492

[13] A. PAZY, Semigroups of Linear Operators and Applications to Partial Differential Equations, Springer Verlag, New York (1983)

[14] C. Preda, P. Preda, Comportări asimptotice ale proceselor evolutive, Ed. Mirton (2007)

[15] P. Preda, A. Pogan, C. Preda, Functionals on function and sequence spaces connected with the exponential stability of evolutionary processes, Czechoslovak Math. J. 56 (131) (2006) 425-435

[16] S. Rolewicz, On uniform N-equistability, J. Math. Anal. Appl. 115 (1986) 434-441

[17] C. Stoica, M. Megan, On asymptotic behaviors of evolution operators in Banach spaces, Ser. Preprint. Mat., Univ. Timişoara 5 (2006) 1-22 